\title{
Association of dietary intake of branched-chain amino acids with long-term risks of CVD, cancer and all-cause mortality
}

\author{
Binbin $\mathrm{Xu}^{1}$, Meng Wang ${ }^{2}$, Liyuan $\mathrm{Pu}^{2}$, Chang Shu ${ }^{3}$, Lian $\mathrm{Li}^{2, *}$ and Liyuan $\mathrm{Han}^{2, *}$ \\ 'Department of Nutrition, HwaMei Hospital, University of Chinese Academy of Sciences, Ningbo, Zhejiang, People's \\ Republic of China: ${ }^{2}$ Department of Global Health, Ningbo Institute of Life and Health Industry, University of Chinese \\ Academy of Sciences, Ningbo, Zhejiang 153000, People's Republic of China: ${ }^{3}$ Tianjin Key Laboratory of Cerebral \\ Vascular and Neurodegenerative Diseases, Tianjin Neurosurgical Institute, Tianjin Huanhu Hospital, Tianjin, People's \\ Republic of China
}

Submitted 26 September 2021: Final revision received 13 December 2021: Accepted 16 December 2021: First published online 21 December 2021

\begin{abstract}
Objectives: We aimed to investigate the associations between dietary branchedchain amino acids (BCAA) intake and long-term risks of CVD, cancer and all-cause mortality in nationwide survey participants aged $\geq 18$.

Design: This was a prospective cohort study. Dietary intakes of BCAA (leucine, isoleucine and valine) were determined from the total nutrient intake document. The main outcomes were CVD, cancer and all-cause mortality.

Setting: A nationally representative sample of US adults were recruited by the National Center for Health Statistics (NCHS) from 1988 to 1994.

Participants: A total of 14397 adults aged $\geq 18$ who participated in the United States National Health and Nutrition Examination Survey III (NHANES III) were included. Results: During 289406 person-years of follow-up, we identified 4219 deaths, including 1133 from CVD and 926 from cancer. After multivariate adjustment, the hazard ratios (95\% confidence intervals) of all-cause mortality in the highest dietary BCAA and isoleucine intake quintile (reference: lowest quintiles) were $0.68(0.48,0.97)$ and $0.68(0.48,0.97)$, respectively. Each one-standard-deviation increase in total dietary BCAA or isoleucine intake was associated with an $18 \%$ or $21 \%$ decrease in the risk of all-cause mortality, respectively. The serum triglyceride (TAG) concentration was found to modify the association between the dietary BCAA intake and allcause mortality $\left(P_{\text {for interaction }}=0.008\right)$.

Conclusions: In a nationally representative cohort, higher dietary intakes of BCAA and isoleucine were independently associated with a lower risk of all-cause mortality, and these associations were stronger in participants with higher serum TAG concentrations.
\end{abstract}

Keywords
Branched-chain amino acids
CVD
Cancer
All-cause mortality

Branched-chain amino acids (BCAA), namely leucine, isoleucine and valine, are an important class of essential amino acids $^{(1)}$. BCAA are comparatively abundant in dietary proteins ${ }^{(2)}$, particularly those in meat, fish, dairy products and eggs ${ }^{(3)}$.

The diet is the only source of BCAA, and a BCAA-rich diet was shown to be positively associated with metabolic health, particularly body-weight management and muscle protein synthesis ${ }^{(4)}$. According to the recommendations of the US Food and Nutrition Commission, the recommended daily intake of leucine, isoleucine and valine for adults is

Binbin Xu and Meng Wang contributed equally to this work.
42, 19 and $24 \mathrm{mg} / \mathrm{kg}$, respectively ${ }^{(5)}$. Adequate intake of BCAA is necessary for protein synthesis and maintaining long-term energy balance ${ }^{(6)}$. Similarly, compelling evidence was reported on the benefits of BCAA supplementation and high dietary intake of BCAA-rich proteins ${ }^{(7)}$. However, the relationship between dietary BCAA intake and CVD, cancer and all-cause mortality in healthy people from the general population has not been determined conclusively.

Given this context, the aim of the current study was to investigate the relationship between dietary BCAA intake and CVD, cancer and all-cause mortality in a large, nationally representative sample of non-institutionalised adults in the USA. 


\section{Materials and Methods}

\section{Study population}

The NHANES III was a large-scale, multi-stage, ongoing, nationally representative survey of a non-institutionalised population sample in the USA and was conducted by the National Center for Health Statistics from 1988 to 1994. Details of the survey design and collection procedures were reported previously ${ }^{(8)}$. The survey included a household interview, physical examination, 24-h dietary recall and blood draw ${ }^{(8)}$. The study was approved by the National Center for Health Statistics Institutional Review Board. All participants provided signed written informed consent.

We included data on participants aged $\geq 18$ years (i.e. adults) from the NHANES III 1988-1994 data set. Those with missing BCAA data and a history of CVD and cancer were excluded from the current study. The flowchart of study participants is shown in online Supplemental Figure 1.

\section{Dietary intake of branched-chain amino acids}

Information on the dietary intake of BCAA was obtained from the NHANES III total nutrient intake document, based on data from the University of Minnesota Nutrition Coordinating Center nutrient database. This database contains information on more than eighty nutrients and food ingredients, including individual fatty acids, amino acids and vitamin $\mathrm{D}^{(9,10)}$. Report the food and beverages (except regular drinking water) consumed by participants in the past $24 \mathrm{~h}$ (from midnight to midnight). The NHANES III Dietary Data Collection system was used to collect all NHANES III dietary recall data ${ }^{(9,10)}$. The Dietary Data Collection system is a computer-based automatic dietary interview and coding system developed by Nutrition Coordinating Center for investigation. In this document, the total nutrient intake of participants whose interview status is complete and reliable was reported. Two US Department of Agriculture food nutrient content databases were used to assign the nutrient value of the NHANES III dietary recalls ${ }^{(9,10)}$. All final editing and determination of the completeness and reliability of the dietary recall are done by National Center for Health Statistics.

\section{Outcome assessment}

The outcome was all-cause mortality. Follow-up data on all-cause, CVD and cancer mortality were ascertained primarily through linked mortality files made publicly available by National Center for Health Statistics. Mortality outcomes were followed until 31 December 2015. Causes of death were determined using the International Classification of Diseases, Tenth Revision. Cardiovascular and cancer deaths were classified using International Classification of Diseases, Tenth Revision codes I00-I78 and C00-C97, respectively.

\section{Covariate assessment}

Information on age, sex, race/ethnicity, marital status, education level, annual household income, smoking and alcohol consumption status and physical activity were collected during interviews, using standardised questionnaires. Participants were divided by race/ethnicity into the following categories: Non-Hispanic White, Non-Hispanic Black, Mexican-American or Other. They were also categorised by marital status as married (married and living as married), widowed or divorced, or single (never married and separated) and by education as less than high school, high school graduate or greater than high school. Annual household incomes were categorised as < US\$20 000, \$20 000$\$ 50000$ or $>\$ 50000$. Physical activity was categorised as poor, intermediate or ideal, as defined in a previous study $^{(11)}$. Smoking and alcohol consumption were categorised as never, former or current.

Serum biochemical profiles were measured at the Lipoprotein Analytical Laboratory at Johns Hopkins University, Baltimore, Maryland, using standardised methods formulated by the US Centers for Disease Control. Type 2 diabetes mellitus was self-reported according to a physician's diagnosis and was diagnosed if a random measure of serum glucose concentration met a certain threshold $(\geq 7 \cdot 1$ $\mathrm{mmol} / \mathrm{l}$ ), or inferred from a participant's use of diabetic medications. Measurements of height, weight and blood pressure were performed following a standardised protocol. BMI was calculated as weight in kilograms divided by height in metres squared. Hypertension was selfreported according to a physician's diagnosis and was diagnosed if systolic blood pressure or diastolic blood pressure met certain thresholds $(\geq 140 \mathrm{mmHg}$ or $\geq 90 \mathrm{mmHg}$, respectively), or inferred from a participant's use of hypertensive medications. Dyslipidaemia was defined as having a physician's diagnosis, currently taking cholesterol-lowering medications, or having a serum TAG concentration > $150 \mathrm{mg} / \mathrm{dl}$ or HDL-cholesterol concentration $<40 \mathrm{mg} / \mathrm{dl}$, based on recommendations by the National Cholesterol Education Program Adult Treatment Panel III.

\section{Statistical analysis}

All participants were classified according to dietary BCAA intake quintiles $(<7 \cdot 8,7 \cdot 8-10 \cdot 9,10 \cdot 9-14 \cdot 1,14 \cdot 1-19$ and $\geq$ $19 \mathrm{~g})$ and baseline demographics, characteristics and clinical variables were compared between the quintiles. Qualitative variables were compared using a $\chi^{2}$ or Fisher's exact test and are reported as frequencies and proportions. Quantitative variables were compared using Student's $t$ test or the Mann-Whitney $U$ test and are reported as medians or means. Tests for linear trends in baseline characteristics across BCAA quintiles were performed using an ANCOVA for continuous variables and the Cochran-Armitage trend test for categorical variables.

Total dietary BCAA intake was calculated by summing the dietary intakes of leucine, isoleucine and valine. 
Total dietary BCAA intake values were log10-transformed before analysis, and hazard ratios (HR) with $95 \%$ CI were calculated for each one-standard-deviation (1-SD) increment in dietary BCAA intake. Spearman correlation coefficients between dietary BCAA and animal protein and vegetable protein intakes were calculated and adjusted for age and BMI.

Associations of total and individual BCAA intakes with all-cause, CVD and cancer mortality were investigated using weighted Cox proportional hazard regression models with the following covariates: age and sex (model 1); model 1 plus animal protein intake (model 2); model 2 plus type 2 diabetes mellitus (model 3); model 4 plus race, marital status, education and annual household income; physical activity; smoking and alcohol consumption status; systolic blood pressure (SBP) and diastolic blood pressure (DBP), BMI; and serum TAG, HDL-cholesterol, LDLcholesterol (LDL), urinary creatinine and uric acid concentrations. Cox proportional hazards models were constructed to estimate survival free from CVD, cancer and all-cause mortality. Proportional hazards assumptions were assessed by analysing Schoenfeld residuals.

In stratified analyses, we assessed the potential modifying effects of the following variables on the association between BCAA intake and outcomes: age $(<60$ or $\geq 60$ years), sex (men or women), BMI $\left(<25\right.$ or $\left.\geq 25 \mathrm{~kg} / \mathrm{m}^{2}\right)$, total serum cholesterol (TC) concentration $(<200$ or $\geq$ $200 \mathrm{mg} / \mathrm{dl}$ ), serum TAG concentration $(<150$ or $\geq 150$ $\mathrm{mg} / \mathrm{dl}$ ), serum HDL concentration (men, $<40$ or $\geq 40$ $\mathrm{mg} / \mathrm{dl}$; women, $<50$ or $\geq 50 \mathrm{mg} / \mathrm{dl}$ ), hypertension (Yes or No) and type 2 diabetes mellitus (Yes or No). Interactions were analysed using the likelihood ratio test with and without the cross-product interaction term and by adjusting for the variables in model 4 . We excluded outcome events that occurred within the 2-year follow-up period, to assess whether the results had been influenced by reverse causation. We used restricted cubic splines based on Cox proportional hazards models to explore the linear relationship between dietary BCAA intake and mortality, with four knots (at the 5th, 35th, 65th and 95th percentiles) ${ }^{(12)}$

In all statistical analyses, sample weights, strata and primary sampling units embedded in the NHANES data were used to account for the complex, multistage, stratified and cluster-sampling design of NHANES (including its oversampling of certain subpopulations). All statistical analyses were conducted using survey modules in SAS software, version 9.4 (SAS Institute, Cary). Two-sided $P$-values of $<0.05$ were regarded as indicating statistical significance.

\section{Results}

\section{Baseline characteristics}

The study population comprised the 14397 adults in the NHANES III database (see online supplementary material,
Supplemental Figure 1). Table 1 depicts differences in the baseline characteristics of participants in different BCAA quintiles. At baseline, statistically significant differences were observed across the BCAA quintiles, in terms of age; BMI; SBP; DBP; serum concentrations of TC, TAG, uric acid, urinary creatinine, LDL and HDL and animal protein intake. Compared with those in the lowest quintiles, participants in the highest quintiles were more likely to be men, Mexican-American, educated beyond high school, current smokers, current alcohol drinkers, unmarried, physically active, to have higher serum TAG concentrations, and to have a higher animal protein intake. Dietary BCAA intake was highly correlated with animal protein intake (Spearman correlation $=0.93$ ) but only weakly correlated with plant protein intake (Spearman correlation $=0.64$ ).

\section{Long-term follow-up}

During 289406 person-years of follow-up, we identified 4219 deaths, including 1133 from CVD and 926 from cancer. The multivariable-adjusted associations of total BCAA, leucine, isoleucine and valine intakes with all-cause mortality are shown in Table 2 and Figure 1. Using model 4 and comparing the highest and lowest quintiles, we determined that intakes of total BCAA (HR: 0.68; $95 \%$ CI: 0.48, 0.97) and isoleucine (HR: 0.68; $95 \% \mathrm{CI}: 0.48,0.97)$ were independently associated with all-cause mortality, whereas no such association was observed for leucine (HR: 0.76; $95 \% \mathrm{CI}: 0.51,1 \cdot 14)$ or valine intake (HR: 0.77; $95 \% \mathrm{CI}$ : $0.55,1.09$ ) (Table 2 and Fig. 1). All tests for linear trends across increasing quintiles were significant $(P<0.05)$. When assessed as a continuous exposure, each 1-SD increase in the total BCAA, isoleucine, leucine or valine intake was associated with an 18\% (HR: 0.82; 95\% CI: $0.73,0.92), 21 \%$ (HR: 0.79 ; $95 \%$ CI: $0.71,0.89$ ), $17 \%$ (HR: 0.83 ; $95 \%$ CI: 0.74 , 0.93), or $18 \%$ (HR: $0.82 ; 95 \%$ CI: $0.73,0.93)$ decrease in the risk of all-cause mortality, respectively.

The multivariable-adjusted associations of total BCAA, leucine, isoleucine and valine intake with CVD mortality are shown in Table 3 and Fig. 1. Using model 4 and comparing the highest and lowest quintiles, we determined that the intakes of total BCAA (HR: 0.57; $95 \%$ CI: 0.29, 1.12), isoleucine (HR: 0.55; 95\% CI: 0.28, 1.10), leucine (HR: 0.65; $95 \% \mathrm{CI}: 0.33,1.29)$ and valine (HR: $0.70 ; 95 \% \mathrm{CI}: 0.37$, 1.34) were not independently associated with CVD mortality (Table 3 and Fig. 1). When assessed as a continuous exposure, each 1-SD increase in the log10-transformed dietary total BCAA, isoleucine, leucine or valine intake was independently associated with a $15 \%$ (HR: 0.85; $95 \%$ CI: $0.72,0.99$ ), $19 \%$ (HR: 0.81; $95 \%$ CI: 0.65, 1.00), $17 \%$ (HR: $0.83 ; 95 \% \mathrm{CI}: 0.68,1.00)$ or $16 \%$ (HR: 0.84 ; $95 \%$ CI: $0 \cdot 71,1 \cdot 00)$ decrease in the risk of CVD mortality, respectively.

The multivariable-adjusted associations of total BCAA, leucine, isoleucine and valine intake with cancer mortality 
Table 1 Baseline characteristics according to quintiles of dietary intake of branched chain amino acids levels

\begin{tabular}{|c|c|c|c|c|c|c|c|c|c|c|c|}
\hline & \multicolumn{2}{|c|}{ Q1 } & \multicolumn{2}{|c|}{ Q2 } & \multicolumn{2}{|c|}{ Q3 } & \multicolumn{2}{|c|}{ Q4 } & \multicolumn{2}{|c|}{ Q5 } & \multirow[b]{2}{*}{$P$-trend } \\
\hline & Mean & SD & Mean & SD & Mean & SD & Mean & SD & Mean & SD & \\
\hline \multicolumn{12}{|c|}{ Continuous variables, mean (SD) } \\
\hline Age (years) & $47 \cdot 15$ & $20 \cdot 11$ & $46 \cdot 23$ & 18.99 & $44 \cdot 30$ & 18.49 & $41 \cdot 60$ & $17 \cdot 03$ & 37.07 & $15 \cdot 02$ & $<0.001$ \\
\hline BMI $\left(\mathrm{kg} / \mathrm{m}^{2}\right)$ & $27 \cdot 14$ & 5.99 & $27 \cdot 12$ & 5.94 & 27.04 & 6.04 & $26 \cdot 84$ & $5 \cdot 62$ & 26.69 & $5 \cdot 72$ & 0.001 \\
\hline $\mathrm{SBP}(\mathrm{mg} / \mathrm{dl})$ & $125 \cdot 40$ & $21 \cdot 22$ & 124.04 & $19 \cdot 54$ & $123 \cdot 16$ & 19.07 & $122 \cdot 19$ & $17 \cdot 90$ & $121 \cdot 13$ & $15 \cdot 37$ & $<0.001$ \\
\hline $\mathrm{DBP}(\mathrm{mg} / \mathrm{dl})$ & 73.35 & $10 \cdot 83$ & 73.54 & $10 \cdot 61$ & 73.75 & 10.64 & 73.94 & $10 \cdot 75$ & 74.57 & $10 \cdot 68$ & $<0.001$ \\
\hline $\mathrm{TC}(\mathrm{mg} / \mathrm{dl})$ & 205.45 & $46 \cdot 90$ & 204.43 & $45 \cdot 62$ & $202 \cdot 79$ & 42.97 & $200 \cdot 13$ & 44.00 & $197 \cdot 74$ & $42 \cdot 63$ & $<0.001$ \\
\hline TAG (mg/dl) & $135 \cdot 68$ & $98 \cdot 88$ & $137 \cdot 25$ & $103 \cdot 77$ & $136 \cdot 10$ & $100 \cdot 90$ & $142 \cdot 61$ & 113.83 & 143.09 & $138 \cdot 79$ & 0.005 \\
\hline $\mathrm{UA}(\mathrm{mg} / \mathrm{dl})$ & $5 \cdot 15$ & 1.51 & 5.08 & 1.45 & $5 \cdot 19$ & 1.46 & 5.37 & 1.43 & 5.60 & 1.43 & $<0.001$ \\
\hline URP (mg/dl) & 143.95 & $94 \cdot 15$ & 131.67 & $82 \cdot 20$ & 134.04 & 84.95 & $136 \cdot 51$ & $79 \cdot 85$ & 147.09 & 81.66 & 0.049 \\
\hline $\mathrm{LDL}(\mathrm{mg} / \mathrm{dl})$ & 127.60 & $40 \cdot 69$ & $125 \cdot 87$ & 39.50 & 127.09 & 37.58 & 123.20 & $37 \cdot 21$ & 122.69 & 37.81 & $<0.001$ \\
\hline $\mathrm{HDL}(\mathrm{mg} / \mathrm{dl})$ & 52.68 & $15 \cdot 63$ & $52 \cdot 14$ & $15 \cdot 96$ & 51.79 & $15 \cdot 75$ & 50.61 & $15 \cdot 31$ & $49 \cdot 70$ & 14.58 & $<0.001$ \\
\hline GLU (mg/dl) & 98.86 & 35.09 & $98 \cdot 71$ & 34.83 & 97.69 & $32 \cdot 64$ & $98 \cdot 24$ & 34.55 & $98 \cdot 17$ & $36 \cdot 23$ & 0.403 \\
\hline Animal Protein $(\mathrm{g} / \mathrm{d})$ & $18 \cdot 87$ & $8 \cdot 60$ & $34 \cdot 24$ & $8 \cdot 60$ & $47 \cdot 23$ & $10 \cdot 31$ & 63.78 & $13 \cdot 77$ & $109 \cdot 20$ & 41.32 & $<0.001$ \\
\hline Categorical variables & $n$ & $\%$ & $n$ & $\%$ & $n$ & $\%$ & $n$ & $\%$ & $n$ & $\%$ & \\
\hline Sex (men) & 718 & $26 \cdot 07$ & 960 & 33.95 & 1154 & 41.65 & 1562 & $55 \cdot 23$ & 2097 & $74 \cdot 10$ & $<0.001$ \\
\hline Type 2 diabetes & 264 & 9.59 & 282 & $9 \cdot 97$ & 261 & $9 \cdot 42$ & 255 & $9 \cdot 02$ & 193 & 6.82 & $<0.001$ \\
\hline \multicolumn{12}{|l|}{ Marital status } \\
\hline Married & 1428 & 51.85 & 1672 & $59 \cdot 12$ & 1678 & $60 \cdot 56$ & 1820 & $64 \cdot 36$ & 1724 & 60.92 & $<0.001$ \\
\hline Widowed/Divorced & 619 & 22.48 & 494 & 17.47 & 444 & $16 \cdot 02$ & 304 & $10 \cdot 75$ & 247 & 8.73 & \\
\hline Never married & 707 & $25 \cdot 67$ & 662 & 23.41 & 649 & 23.42 & 704 & 24.89 & 859 & $30 \cdot 35$ & \\
\hline \multicolumn{12}{|l|}{ Race/ethnicity } \\
\hline Non-Hispanic White & 999 & $36 \cdot 27$ & 1087 & 38.44 & 1061 & 38.29 & 1072 & 37.91 & 923 & $32 \cdot 61$ & $<0.001$ \\
\hline Non-Hispanic Black & 952 & 34.57 & 842 & $29 \cdot 77$ & 770 & $27 \cdot 79$ & 711 & $25 \cdot 14$ & 849 & $30 \cdot 00$ & \\
\hline Mexican-American & 706 & $25 \cdot 64$ & 793 & 28.04 & 827 & 29.84 & 915 & $32 \cdot 36$ & 925 & 32.69 & \\
\hline Other & 97 & 3.52 & 106 & 3.75 & 113 & 4.08 & 130 & $4 \cdot 60$ & 133 & $4 \cdot 70$ & \\
\hline \multicolumn{12}{|l|}{ Education } \\
\hline Less than high school & 1266 & 45.97 & 1154 & $40 \cdot 81$ & 1014 & 36.59 & 1062 & 37.55 & 1019 & 36.01 & $<0.001$ \\
\hline High school graduate & 867 & 31.48 & 930 & 32.89 & 900 & 32.48 & 866 & $30 \cdot 62$ & 929 & $32 \cdot 83$ & \\
\hline Greater than high school & 621 & $22 \cdot 55$ & 744 & $26 \cdot 31$ & 857 & 30.93 & 900 & $31 \cdot 82$ & 882 & $31 \cdot 17$ & \\
\hline \multicolumn{12}{|l|}{ Annual household income } \\
\hline$<\$ 20000$ & 1379 & $50 \cdot 07$ & 1240 & 43.85 & 1116 & $40 \cdot 27$ & 1140 & $40 \cdot 31$ & 1181 & 41.73 & $<0.001$ \\
\hline$\$ 20000-\$ 50000$ & 891 & $32 \cdot 35$ & 1005 & $35 \cdot 54$ & 1052 & 37.96 & 1046 & 36.99 & 1075 & 37.99 & \\
\hline$>\$ 50000$ & 484 & $17 \cdot 57$ & 583 & $20 \cdot 62$ & 603 & $21 \cdot 76$ & 642 & $22 \cdot 70$ & 574 & $20 \cdot 28$ & \\
\hline \multicolumn{12}{|l|}{ Drinking } \\
\hline Never & 1977 & 71.97 & 1958 & $69 \cdot 33$ & 1763 & 63.78 & 1664 & $59 \cdot 01$ & 1457 & 51.59 & $<0.001$ \\
\hline Former & 403 & 14.67 & 438 & $15 \cdot 51$ & 499 & 18.05 & 539 & $19 \cdot 11$ & 627 & $22 \cdot 20$ & \\
\hline Current & 367 & $13 \cdot 36$ & 428 & $15 \cdot 16$ & 502 & $18 \cdot 16$ & 617 & $21 \cdot 88$ & 740 & $26 \cdot 20$ & \\
\hline \multicolumn{12}{|l|}{ Smoking } \\
\hline Never & 1521 & $55 \cdot 25$ & 1509 & $53 \cdot 36$ & 1404 & $50 \cdot 67$ & 1318 & $46 \cdot 61$ & 1261 & 44.59 & $<0.001$ \\
\hline Former & 502 & $18 \cdot 23$ & 626 & $22 \cdot 14$ & 617 & $22 \cdot 27$ & 700 & $24 \cdot 75$ & 617 & 21.82 & \\
\hline Current & 730 & $26 \cdot 52$ & 693 & $24 \cdot 50$ & 750 & $27 \cdot 07$ & 810 & $28 \cdot 64$ & 950 & 33.59 & \\
\hline \multicolumn{12}{|l|}{ Physical activity } \\
\hline Poor & 671 & $24 \cdot 36$ & 645 & $22 \cdot 81$ & 555 & $20 \cdot 03$ & 502 & $17 \cdot 75$ & 451 & 15.94 & $<0.001$ \\
\hline Intermediate & 1277 & $46 \cdot 37$ & 1371 & 48.48 & 1397 & $50 \cdot 42$ & 1474 & $52 \cdot 12$ & 1443 & 50.99 & \\
\hline Ideal & 806 & $29 \cdot 27$ & 812 & $28 \cdot 71$ & 819 & 29.56 & 852 & $30 \cdot 13$ & 936 & 33.07 & \\
\hline
\end{tabular}

SBP, systolic blood pressure; DBP, diastolic blood pressure; TC, total cholesterol, UA, uric acid; URP, urinary creatinine; GLU, glucose.

are shown in Table 4 and Fig. 1. Using model 4 and comparing the highest and lowest quintiles, we determined that the intakes of total BCAA (HR: $0.73 ; 95 \%$ CI: 0.32, 1.69), isoleucine (HR: 0.54; $95 \%$ CI: 0.21, 1.38), leucine (HR: 0.69; 95\% CI: $0.31,1.56$ ) and valine (HR: $0.85 ; 95 \%$ CI: 0.38 , 1.90) were not independently associated with cancer mortality (Table 4 and Fig. 1). Similarly, when assessed as a continuous exposure, we found that each 1-SD increase in the log10-transformed dietary intake of total and individual BCAA was not significantly associated with the risk of cancer mortality. Multivariable-adjusted spline regression models showed a negative linear dose-response association between dietary BCAA intake and all-cause mortality $\left(P_{\text {for linearity }}=0.001, P_{\text {for nonlinearity }}=0.964 ;\right.$ Fig. 2$)$.

\section{Subgroups analyses}

In stratified analyses, after adjusting for potential risk factors we found that the association between dietary BCAA intake and all-cause mortality was modified by serum TAG concentration. Specifically, a higher dietary BCAA intake was associated with a decreased risk of all-cause 
Table 2 Hazard ratios $(95 \% \mathrm{Cl})$ for risk of all-cause mortality according to quintiles of branched chain amino acids (BCAA)

\begin{tabular}{|c|c|c|c|c|c|c|c|c|c|c|c|c|}
\hline & \multirow[b]{2}{*}{ Q1 } & \multicolumn{2}{|c|}{ Q2 } & \multicolumn{2}{|c|}{ Q3 } & \multicolumn{2}{|c|}{ Q4 } & \multicolumn{2}{|c|}{ Q5 } & \multirow[b]{2}{*}{$P$-trend } & \multicolumn{2}{|c|}{ Each SD } \\
\hline & & Hazard ratios & $95 \% \mathrm{Cl}$ & Hazard ratios & $95 \% \mathrm{Cl}$ & Hazard ratios & $95 \% \mathrm{Cl}$ & Hazard ratios & $95 \% \mathrm{Cl}$ & & Hazard ratios & $95 \% \mathrm{Cl}$ \\
\hline \multicolumn{13}{|l|}{ Total BCAA } \\
\hline Median (g/d) & 5.92 & $9 \cdot 34$ & & $12 \cdot 34$ & & $16 \cdot 16$ & & 24.05 & & & & \\
\hline N/person-years & $1027 / 54016$ & $953 / 56908$ & & $851 / 57091$ & & 759/59792 & & 629/61599 & & & & \\
\hline Model 1 & 1.00 & 0.81 & $0.71,0.93$ & 0.78 & $0.67,0.91$ & 0.78 & $0.66,0.91$ & 0.85 & $0.71,1.02$ & 0.062 & 0.94 & $0.88,0.99$ \\
\hline Model 2 & 1.00 & 0.76 & $0.66,0.87$ & 0.69 & $0.58,0.83$ & 0.64 & $0.52,0.78$ & 0.58 & $0.44,0.76$ & 0.0001 & 0.77 & $0.72,0.84$ \\
\hline Model 3 & 1.00 & 0.75 & $0.66,0.85$ & 0.68 & $0.58,0.81$ & 0.63 & $0.52,0.76$ & 0.57 & $0.44,0.75$ & $<0.0001$ & 0.77 & $0.72,0.83$ \\
\hline Model 4 & 1.00 & 0.77 & $0.62,0.94$ & 0.78 & $0.64,0.94$ & 0.64 & $0.50,0.83$ & 0.68 & $0.48,0.97$ & 0.006 & 0.82 & $0.73,0.92$ \\
\hline \multicolumn{13}{|l|}{ Isoleucine } \\
\hline Median (g/d) & 1.47 & $2 \cdot 39$ & & $3 \cdot 17$ & & $4 \cdot 19$ & & $6 \cdot 27$ & & & & \\
\hline $\mathrm{N} /$ person-years & $945 / 50431$ & $946 / 55066$ & & $911 / 61812$ & & $794 / 60768$ & & $623 / 61330$ & & & & \\
\hline Model 1 & 1.00 & 0.83 & $0.73,0.94$ & 0.76 & $0.66,0.87$ & 0.79 & $0.68,0.92$ & 0.84 & $0.70,1.01$ & 0.054 & 0.94 & $0.88,0.99$ \\
\hline Model 2 & 1.00 & 0.77 & $0.68,0.88$ & 0.67 & $0.57,0.79$ & 0.64 & $0.53,0.78$ & 0.55 & $0.42,0.72$ & $<0.0001$ & 0.77 & $0.71,0.83$ \\
\hline Model 3 & 1.00 & 0.76 & $0.68,0.86$ & 0.66 & $0.57,0.78$ & 0.63 & $0.52,0.76$ & 0.55 & $0.42,0.72$ & $<0.0001$ & 0.76 & $0.71,0.82$ \\
\hline Model 4 & 1.00 & 0.80 & $0.64,1.00$ & 0.76 & $0.63,0.91$ & 0.62 & $0.49,0.78$ & 0.68 & $0.48,0.97$ & 0.001 & 0.79 & $0.71,0.89$ \\
\hline \multicolumn{13}{|l|}{ Leucine } \\
\hline Median (g/d) & $2 \cdot 62$ & $4 \cdot 15$ & & 5.49 & & $7 \cdot 20$ & & 10.73 & & & & \\
\hline $\mathrm{N} /$ person-years & $1020 / 52708$ & 949/57052 & & $830 / 56245$ & & 787/61226 & & 633/62176 & & & & \\
\hline Model 1 & 1.00 & 0.83 & $0.71,0.96$ & 0.77 & $0.67,0.89$ & 0.80 & $0.69,0.93$ & 0.89 & $0.73,1.09$ & 0.134 & 0.94 & $0.89,1.00$ \\
\hline Model 2 & 1.00 & 0.79 & $0.68,0.91$ & 0.70 & $0.59,0.83$ & 0.69 & $0.57,0.83$ & 0.66 & $0.50,0.88$ & 0.001 & 0.79 & $0.73,0.85$ \\
\hline Model 3 & 1.00 & 0.78 & $0.68,0.89$ & 0.69 & $0.59,0.81$ & 0.68 & $0.57,0.81$ & 0.66 & $0.49,0.88$ & 0.0004 & 0.78 & $0.73,0.84$ \\
\hline Model 4 & 1.00 & 0.79 & $0.64,0.99$ & 0.81 & $0.67,0.97$ & 0.71 & $0.55,0.92$ & 0.76 & $0.51,1.14$ & 0.038 & 0.83 & $0.74,0.93$ \\
\hline \multicolumn{13}{|l|}{ Valine } \\
\hline Median (g/d) & $1 \cdot 70$ & 2.71 & & 3.57 & & 4.70 & & $7 \cdot 10$ & & & & \\
\hline N/person-years & $1005 / 53204$ & $933 / 55818$ & & $870 / 56267$ & & $753 / 60598$ & & $658 / 63521$ & & & & \\
\hline Model 1 & 1.00 & 0.82 & $0.72,0.95$ & 0.81 & $0.70,0.93$ & 0.75 & $0.64,0.88$ & 0.86 & $0.71,1.03$ & 0.044 & 0.93 & $0.88,0.99$ \\
\hline Model 2 & 1.00 & 0.77 & $0.68,0.89$ & 0.72 & $0.61,0.84$ & 0.63 & $0.51,0.76$ & 0.59 & $0.45,0.78$ & $<0.0001$ & 0.77 & $0.71,0.84$ \\
\hline Model 3 & 1.00 & 0.77 & $0.68,0.87$ & 0.71 & $0.62,0.83$ & 0.62 & $0.51,0.74$ & 0.59 & $0.45,0.76$ & $<0.0001$ & 0.77 & $0.72,0.83$ \\
\hline Model 4 & 1.00 & 0.80 & $0.64,1.00$ & 0.85 & $0.71,1.02$ & 0.66 & $0.51,0.84$ & 0.77 & $0.55,1.09$ & 0.012 & 0.82 & $0.73,0.93$ \\
\hline
\end{tabular}

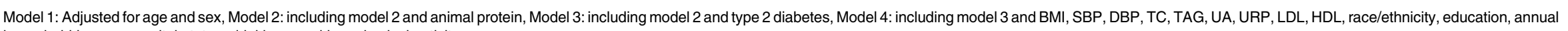

household income, marital status, drinking smoking physical activity. 
A population-based study

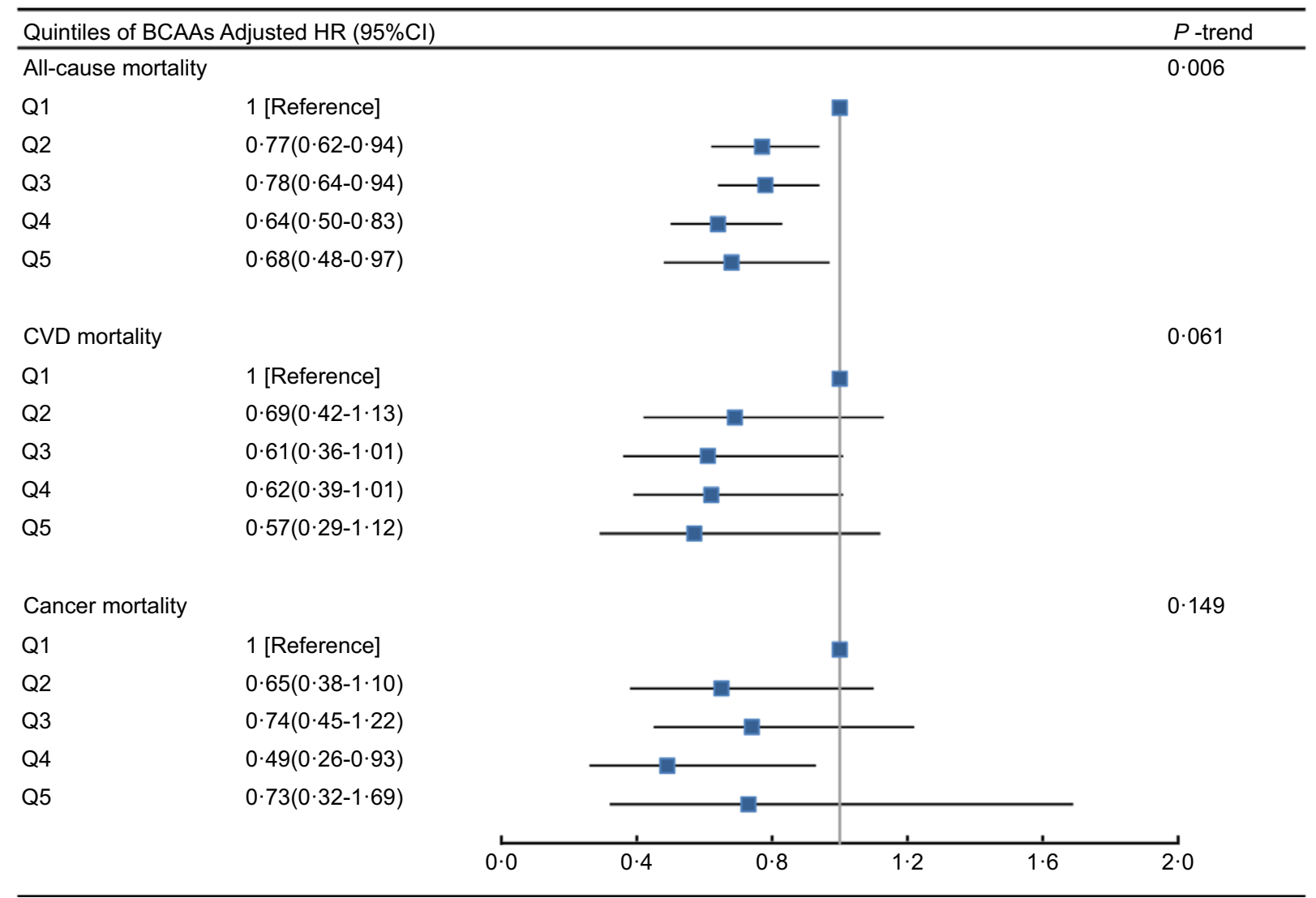

Fig. 1 (colour online) Hazard ratios $(95 \% \mathrm{Cl}$ ) for risk of all-cause, CVD and cancer mortality. According to Quintiles of branched chain amino acids (BCAA) Quintiles of BCAA Adjusted HR $(95 \% \mathrm{Cl})$

mortality in participants with a higher serum TAG concentration; the HR for the highest BCAA intake quintile was 0.43 (95\% CI: 0.22, 0.84; $P_{\text {for interaction }}=0.008$; see online Supplemental Table 1). Other sub-group analysis was shown in Supplemental Tables 2-12.

\section{Sensitivity analysis}

To examine the role of reverse causation, 215 participants whose outcomes occurred during the first 2 years of followup were excluded from the analysis. The results obtained using the remaining sample were similar to those observed in the full sample (data not shown). A sensitivity analysis that excluded participants with type 2 diabetes mellitus yielded similar results for all-cause mortality and CVD mortality (data not shown).

\section{Discussion}

In this large prospective study of a nationally representative cohort, we found after multivariate adjustment that higher dietary intakes of total BCAA and isoleucine were independently associated with a decreased risk of all-cause mortality during long-term follow-up (27 years). Furthermore, these associations were stronger in participants with higher serum TAG concentrations. The trends remained robust in stratified and sensitivity analyses.

Only a few studies have examined the relationship between dietary BCAA intake and all-cause mortality. The findings from the Nurses' Health Study and the Health Professionals Follow-up Study suggested that there were positive associations between a higher intake of dietary BCAA and the risk of all-cause mortality in patients with colorectal cancer, particularly male patients ${ }^{(13)}$. Shah et al. found that higher serum concentrations of BCAA were negatively associated with mortality in patients who underwent cardiac catheterisation ${ }^{(14)}$. In another study of 865 patients, elevated serum BCAA concentrations were associated with increased risks of all-cause mortality and heart failure in patients with acute heart disease ${ }^{(15)}$. However, recent studies have found only a weak correlation between dietary BCAA intake and circulating BCAA concentrations $^{(16,17)}$, and the above-cited studies were conducted in participants with CVD or metabolic diseases. Thus, further research is needed to better understand the correlation between dietary BCAA intake and all-cause mortality in different populations.

In a cohort of older community-dwelling males, lower serum BCAA concentrations were associated with increases in mortality and major cardiovascular endpoints 
Table 3 Hazard ratios $(95 \% \mathrm{Cl}$ ) for risk of CVD mortality according to quintiles of BCAA

\begin{tabular}{|c|c|c|c|c|c|c|c|c|c|c|c|c|}
\hline & \multirow[b]{2}{*}{ Q1 } & \multicolumn{2}{|c|}{ Q2 } & \multicolumn{2}{|c|}{ Q3 } & \multicolumn{2}{|c|}{ Q4 } & \multicolumn{2}{|c|}{ Q5 } & \multirow[b]{2}{*}{$P$-trend } & \multicolumn{2}{|c|}{ Each SD } \\
\hline & & Hazard ratios & $95 \% \mathrm{Cl}$ & Hazard ratios & $95 \% \mathrm{Cl}$ & Hazard ratios & $95 \% \mathrm{Cl}$ & Hazard ratios & $95 \% \mathrm{Cl}$ & & Hazard ratios & $95 \% \mathrm{Cl}$ \\
\hline \multicolumn{13}{|l|}{ Total BCAA } \\
\hline Median $(\mathrm{g} / \mathrm{d})$ & 5.92 & $9 \cdot 34$ & & $12 \cdot 34$ & & $16 \cdot 16$ & & 24.05 & & & & \\
\hline N/person-years & $299 / 54016$ & $260 / 56908$ & & $244 / 57092$ & & $180 / 59793$ & & $150 / 61599$ & & & & \\
\hline Model 1 & 1.00 & 0.94 & $0.69,1.27$ & 0.71 & $0.56,0.91$ & 0.67 & $0.48,0.94$ & 0.70 & $0.49,0.99$ & 0.004 & 0.90 & $0.80,1.00$ \\
\hline Model 2 & 1.00 & 0.84 & $0.62,1.13$ & 0.58 & $0.43,0.78$ & 0.50 & $0.32,0.77$ & 0.37 & $0.21,0.66$ & 0.001 & 0.82 & $0.67,0.99$ \\
\hline Model 3 & 1.00 & 0.83 & $0.62,1.11$ & 0.57 & $0.43,0.78$ & 0.50 & $0.32,0.76$ & 0.36 & $0.20,0.65$ & 0.001 & 0.82 & $0.68,0.99$ \\
\hline Model 4 & 1.00 & 0.69 & $0.42,1.13$ & 0.61 & $0.36,1.01$ & 0.62 & $0.39,1.01$ & 0.57 & $0.29,1.12$ & 0.061 & 0.85 & $0.72,0.99$ \\
\hline \multicolumn{13}{|l|}{ Isoleucine } \\
\hline Median $(\mathrm{g} / \mathrm{d})$ & 1.47 & 2.39 & & $3 \cdot 17$ & & $4 \cdot 19$ & & $6 \cdot 27$ & & & & \\
\hline $\mathrm{N} /$ person-years & $274 / 50431$ & $268 / 55066$ & & $252 / 61812$ & & $190 / 60768$ & & $149 / 61330$ & & & & \\
\hline Model 1 & 1.00 & 0.91 & $0.68,1.21$ & 0.71 & $0.54,0.92$ & 0.73 & $0.57,0.94$ & 0.72 & $0.50,1.04$ & 0.009 & 0.90 & $0.81,1.01$ \\
\hline Model 2 & 1.00 & 0.84 & $0.64,1.12$ & 0.61 & $0.46,0.82$ & 0.58 & $0.41,0.82$ & 0.46 & $0.26,0.83$ & 0.001 & 0.82 & $0.66,1.02$ \\
\hline Model 3 & 1.00 & 0.83 & $0.63,1.08$ & 0.61 & $0.45,0.82$ & 0.57 & $0.41,0.81$ & 0.45 & $0.25,0.80$ & 0.001 & 0.82 & $0.66,1.02$ \\
\hline Model 4 & 1.00 & 0.67 & $0.42,1.06$ & 0.59 & $0.36,0.98$ & 0.62 & $0.37,1.04$ & 0.55 & $0.28,1.10$ & 0.083 & 0.81 & $0.65,1.00$ \\
\hline \multicolumn{13}{|l|}{ Leucine } \\
\hline Median (g/d) & $2 \cdot 62$ & $4 \cdot 15$ & & 5.49 & & $7 \cdot 20$ & & $10 \cdot 73$ & & & & \\
\hline $\mathrm{N} /$ person-years & $298 / 52708$ & $255 / 57052$ & & $246 / 56245$ & & $185 / 61226$ & & $149 / 62176$ & & & & \\
\hline Model 1 & 1.00 & 0.95 & $0.68,1.31$ & 0.71 & $0.56,0.91$ & 0.66 & $0.49,0.90$ & 0.74 & $0.53,1.05$ & 0.007 & 0.90 & $0.80,1.01$ \\
\hline Model 2 & 1.00 & 0.87 & $0.63,1.20$ & 0.61 & $0.44,0.84$ & 0.53 & $0.34,0.83$ & 0.45 & $0.23,0.89$ & 0.003 & 0.82 & $0.66,1.01$ \\
\hline Model 3 & 1.00 & 0.86 & $0.62,1.18$ & 0.60 & $0.44,0.83$ & 0.53 & $0.34,0.82$ & 0.45 & $0.22,0.89$ & 0.003 & 0.82 & $0.66,1.01$ \\
\hline Model 4 & 1.00 & 0.76 & $0.43,1.34$ & 0.64 & $0.40,1.01$ & 0.61 & $0.38,0.97$ & 0.65 & $0.33,1.29$ & 0.031 & 0.83 & $0.68,1.00$ \\
\hline \multicolumn{13}{|l|}{ Valine } \\
\hline Median (g/d) & $1 \cdot 70$ & $2 \cdot 71$ & & 3.57 & & 4.70 & & $7 \cdot 10$ & & & & \\
\hline N/person-years & $281 / 53204$ & $268 / 55818$ & & $248 / 56267$ & & $180 / 50598$ & & $156 / 63521$ & & & & \\
\hline Model 1 & 1.00 & 0.98 & $0.72,1.34$ & 0.73 & $0.58,0.93$ & 0.69 & $0.50,0.94$ & 0.75 & $0.53,1.07$ & 0.009 & 0.90 & $0.80,1.01$ \\
\hline Model 2 & 1.00 & 0.92 & $0.68,1.25$ & 0.64 & $0.48,0.86$ & 0.57 & $0.37,0.87$ & 0.50 & $0.27,0.94$ & 0.003 & 0.82 & $0.66,1.01$ \\
\hline Model 3 & 1.00 & 0.92 & $0.68,1.24$ & 0.64 & $0.48,0.86$ & 0.57 & $0.37,0.87$ & 0.50 & $0.27,0.94$ & 0.003 & 0.82 & $0.66,1.00$ \\
\hline Model 4 & 1.00 & 0.88 & $0.51,1.54$ & 0.70 & $0.44,1.10$ & 0.73 & $0.46,1.17$ & 0.70 & $0.37,1.34$ & 0.128 & 0.84 & $0.71,1.00$ \\
\hline
\end{tabular}

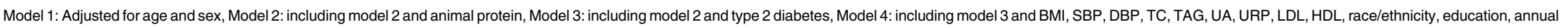
household income, marital status, drinking, smoking, physical activity. 
Table 4 Hazard ratios $(95 \% \mathrm{Cl})$ for risk of cancer mortality according to quintiles of BCAA

\begin{tabular}{|c|c|c|c|c|c|c|c|c|c|c|c|c|}
\hline & \multirow[b]{2}{*}{ Q1 } & \multicolumn{2}{|c|}{ Q2 } & \multicolumn{2}{|c|}{ Q3 } & \multicolumn{2}{|c|}{ Q4 } & \multicolumn{2}{|c|}{ Q5 } & \multirow[b]{2}{*}{$P$-trend } & \multicolumn{2}{|c|}{ Each SD } \\
\hline & & Hazard ratios & $95 \% \mathrm{Cl}$ & Hazard ratios & $95 \% \mathrm{Cl}$ & Hazard ratios & $95 \% \mathrm{Cl}$ & Hazard ratios & $95 \% \mathrm{Cl}$ & & Hazard ratios & $95 \% \mathrm{Cl}$ \\
\hline \multicolumn{13}{|l|}{ Total BCAA } \\
\hline Median (g/d) & 5.92 & 9.34 & & $12 \cdot 34$ & & $16 \cdot 16$ & & 24.05 & & & & \\
\hline $\mathrm{N} /$ person-years & $202 / 54016$ & $233 / 56908$ & & $176 / 57092$ & & $166 / 59793$ & & $149 / 61599$ & & & & \\
\hline Model 1 & 1.00 & 0.86 & $0.67,1 \cdot 12$ & 0.94 & $0.67,1.32$ & 1.03 & $0.75,1.41$ & 1.08 & $0.78,1.48$ & 0.404 & 1.03 & $0.92,1.17$ \\
\hline Model 2 & 1.00 & 0.86 & $0.65,1.12$ & 0.93 & $0.68,1.27$ & 1.00 & $0.67,1.49$ & 1.03 & $0.58,1.84$ & 0.848 & 0.93 & $0.77,1.12$ \\
\hline Model 3 & 1.00 & 0.86 & $0.65,1 \cdot 12$ & 0.92 & $0.68,1.25$ & 1.00 & $0.67,1.48$ & 1.03 & $0.58,1.84$ & 0.858 & 0.92 & $0.77,1 \cdot 10$ \\
\hline Model 4 & 1.00 & 0.65 & $0.38,1 \cdot 10$ & 0.74 & $0.45,1.22$ & 0.49 & $0.26,0.93$ & 0.73 & $0.32,1.69$ & 0.149 & 0.81 & $0.56,1.18$ \\
\hline \multicolumn{13}{|l|}{ Isoleucine } \\
\hline Median (g/d) & 1.47 & 2.39 & & 3.17 & & 4.19 & & $6 \cdot 27$ & & & & \\
\hline N/person-years & $189 / 50431$ & $217 / 55066$ & & $197 / 61812$ & & $173 / 60768$ & & $150 / 61330$ & & & & \\
\hline Model 1 & 1.00 & 0.90 & $0.66,1.22$ & 0.94 & $0.69,1.30$ & $1 \cdot 16$ & $0.87,1.54$ & 1.00 & $0.72,1.39$ & 0.482 & 1.04 & $0.92,1.17$ \\
\hline Model 2 & 1.00 & 0.87 & $0.63,1.20$ & 0.89 & $0.65,1.22$ & 1.04 & $0.68,1.59$ & 0.84 & $0.46,1.51$ & 0.942 & 0.94 & $0.77,1.15$ \\
\hline Model 3 & 1.00 & 0.87 & $0.63,1.20$ & 0.89 & $0.65,1.20$ & 1.04 & $0.68,1.59$ & 0.84 & $0.46,1.52$ & 0.930 & 0.94 & $0.77,1.14$ \\
\hline Model 4 & 1.00 & 0.66 & $0.38,1.17$ & 0.68 & $0.40,1.16$ & 0.50 & $0.26,0.96$ & 0.54 & $0.21,1.38$ & 0.101 & 0.80 & $0.54,1.18$ \\
\hline \multicolumn{13}{|l|}{ Leucine } \\
\hline Median (g/d) & 2.62 & 4.15 & & 5.49 & & $7 \cdot 20$ & & $10 \cdot 73$ & & & & \\
\hline $\mathrm{N} /$ person-years & $202 / 52708$ & $233 / 57052$ & & $167 / 56245$ & & $172 / 61226$ & & $152 / 62176$ & & & & \\
\hline Model 1 & 1.00 & 0.87 & $0.66,1.15$ & 0.94 & $0.66,1.34$ & 1.01 & $0.74,1.39$ & 1.09 & $0.80,1.48$ & 0.389 & 1.03 & $0.91,1.16$ \\
\hline Model 2 & 1.00 & 0.86 & $0.64,1 \cdot 16$ & 0.93 & $0.67,1.29$ & 0.99 & $0.67,1.47$ & 1.05 & $0.61,1.82$ & 0.815 & 0.92 & $0.77,1.10$ \\
\hline Model 3 & 1.00 & 0.86 & $0.64,1.16$ & 0.92 & $0.67,1.27$ & 0.99 & $0.67,1.46$ & 1.05 & $0.61,1.82$ & 0.823 & 0.92 & $0.78,1.09$ \\
\hline Model 4 & 1.00 & 0.62 & $0.37,1.05$ & 0.83 & $0.50,1.40$ & 0.45 & $0.26,0.79$ & 0.69 & $0.31,1.56$ & 0.084 & 0.81 & $0.56,1.17$ \\
\hline \multicolumn{13}{|l|}{ Valine } \\
\hline Median $(\mathrm{g} / \mathrm{d})$ & 1.70 & $2 \cdot 71$ & & 3.57 & & $4 \cdot 70$ & & $7 \cdot 10$ & & & & \\
\hline $\mathrm{N} /$ person-years & 202/53204 & $223 / 55818$ & & $185 / 56267$ & & $163 / 60598$ & & $153 / 63521$ & & & & \\
\hline Model 1 & 1.00 & 0.86 & $0.65,1.14$ & 0.95 & $0.69,1.33$ & 0.97 & $0.71,1.34$ & 1.09 & $0.78,1.51$ & 0.495 & 1.03 & $0.92,1.16$ \\
\hline Model 2 & 1.00 & 0.85 & $0.64,1.13$ & 0.93 & $0.67,1.28$ & 0.92 & $0.61,1.40$ & 1.00 & $0.55,1.82$ & 0.952 & 0.92 & $0.77,1 \cdot 11$ \\
\hline Model 3 & 1.00 & 0.85 & $0.64,1.12$ & 0.92 & $0.67,1.26$ & 0.92 & $0.61,1.37$ & 0.99 & $0.55,1.80$ & 0.930 & 0.92 & $0.77,1.10$ \\
\hline Model 4 & 1.00 & 0.60 & $0.35,1.05$ & 0.79 & $0.49,1.28$ & 0.47 & $0.26,0.86$ & 0.85 & $0.38,1.90$ & 0.216 & 0.85 & $0.60,1.20$ \\
\hline
\end{tabular}

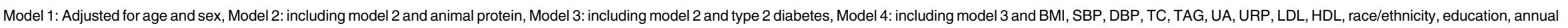

household income, marital status, drinking, smoking, physical activity. 


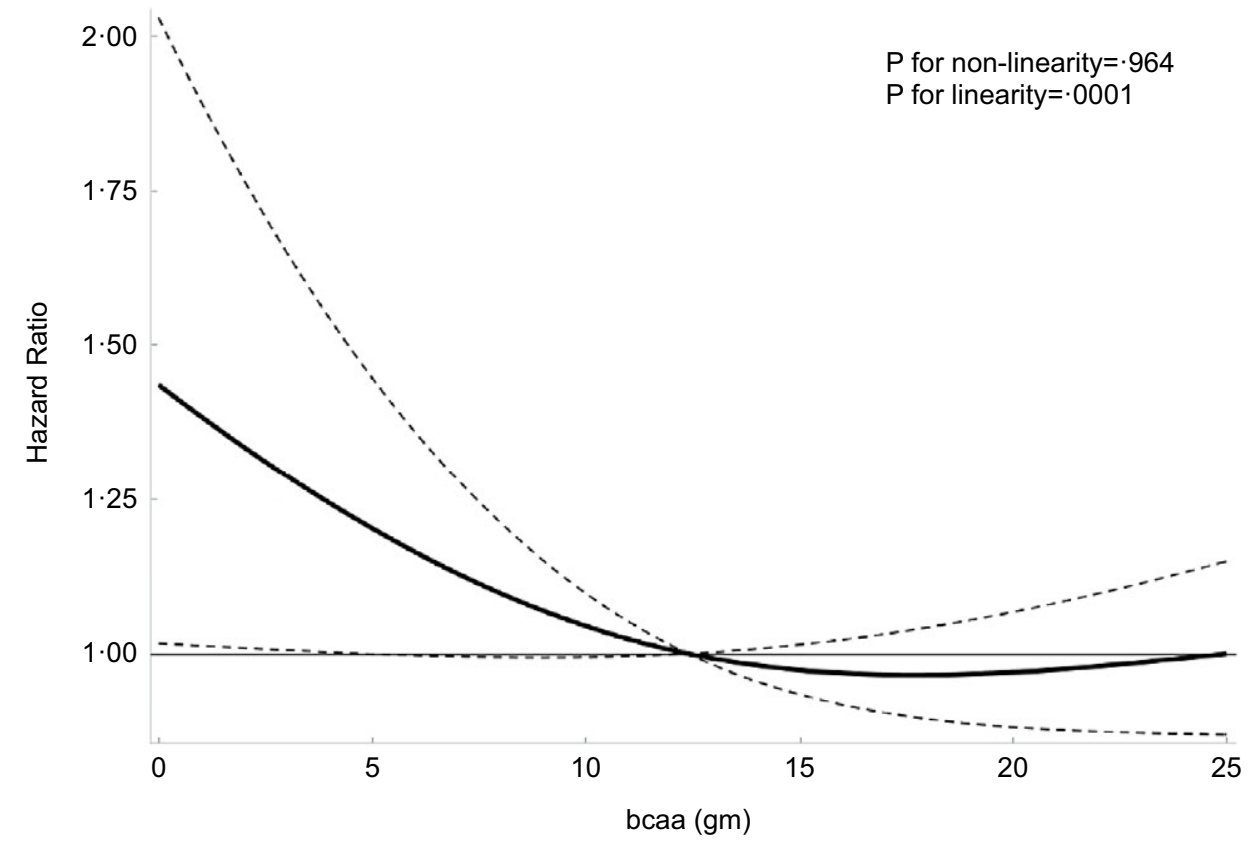

Fig. 2 Association of dietary branched chain amino acids (BCAA). Intake with risk of all-cause mortality. Adjusted hazard ratios (HR) of all-cause mortality according to dietary BCAA intake. HR and $95 \% \mathrm{Cl}$ derived from restricted cubic spline regression, with knots placed at the 5th, 35th, 65th and 95th percentiles of dietary BCAA intake. OR adjusted for the same variables as model 4 in Table 2.

due to increasing age and frailty ${ }^{(18)}$. In the current study, participants in the highest BCAA intake quintiles were younger, and the relationship between BCAA intake and CVD and metabolic disease was more evident in young adults than in older adults ${ }^{(19)}$. Therefore, the effects of potential confounding factors, such as age or frailty, on the association between BCAA intake and disease outcomes should be considered further.

Associations between BCAA intake and disease may also depend on the dietary source of BCAA. The major food sources of BCAA are meat, fish, cereals, dairy products, vegetables and eggs ${ }^{(20)}$. In the current study, dietary BCAA intake was highly correlated with animal protein intake, but only weakly correlated with plant protein intake. Animal and plant proteins differ mainly in their proportions of essential amino acids, including BCAA, which is higher in animal proteins. Plant proteins were reported to contain lower concentrations of essential amino acids (such as leucine, methionine, lysine and tryptophan) than animal proteins $^{(21)}$. However, in the current study, after multivariate adjustment participants with higher animal protein intakes were not found to have a decreased risk of all-cause mortality relative to those with the lowest intake ( $\mathrm{HR}=0.91,95 \%$ CI: $0.72,1.15)$. Therefore, animal protein intake did not explain the negative association between dietary BCAA intake and all-cause mortality in the current study.

There are biologically plausible mechanisms that potentially explain the protective effect of a high BCAA intake against mortality. For example, BCAA can act as a fuel source to decrease protein degradation, a likely consequence of increased mitochondrial biogenesis and reduced oxidative stress in cardiac and skeletal muscles, via endothelial nitric oxide synthase-mediated mechanisms ${ }^{(22)}$. There is also substantial evidence that BCAA supplementation or a BCAA-rich diet has positive effects on body weight regulation, muscle protein synthesis, glucose homeostasis, ageing processes and healthspan exten$\operatorname{sion}^{(23)}$. In addition, adequate BCAA intake is necessary for protein synthesis and maintaining energy balance over the long term ${ }^{(24,25)}$. Furthermore, BCAA supplementation was recommended to reduce sarcopenia in older adults ${ }^{(26)}$ and to improve muscle mass and physical performance in athletes $^{(27)}$.

Interestingly, we observed a stronger association between BCAA intake and mortality in participants with higher serum TAG concentrations. A high dietary intake of BCAA, particularly leucine, was reported to be associated with improvements in dyslipidaemia, pulse-wave velocity, intimal-medial thickness and atherosclerosis development, and these effects were independent of genetic confounders ${ }^{(28)}$. Leucine has been shown to significantly affect macrophage atherogenicity and protect against atherogenesis, mainly by modulating cellular TAG metabolism ${ }^{(29,30)}$. However, elevated plasma BCAA concentrations have been shown to be significantly associated with high serum TAG concentrations in middle-aged and older adults ${ }^{(18,31)}$. Nevertheless, the mechanism underlying the combined effects of BCAA and TAGs on mortality remains unclear, and further research is required to investigate this association. 
To our knowledge, this is the first large prospective study to assess the association of dietary BCAA intake with the risks of all-cause, CVD mortality and cancer mortality in a general US population sample. One strength of our study was its large, nationally representative cohort design with up to 27 years of follow-up, which facilitates generalisation of the findings to the general US population. Other strengths include its use of data collected using standardised procedures with quality control measures and adjustment to control for potentially confounding effects due to various demographic, socio-economic, lifestyle and dietary factors. We found that the protective association of BCAA intake was robust to the exclusion of subjects who died within the first 2 years of follow-up.

However, our study also has some limitations. First, the dietary BCAA intake was collected only once during the follow-up period. The inability to determine changes in BCAA over time may have led to biased results. Second, survey data on household income and physical activity levels, smoking history and dietary intakes were self-reported by participants in the NHANES surveys, which may have contributed to reporting bias. Third, there may have been misclassification of the underlying and contributing causes of death and residual confounding and competing risks for cause-specific mortalities. In addition, although we adjusted for the common risk factors known to be associated with mortality, some uncontrolled and unmeasured confounders might remain. Genetic data were not available in our study, and therefore we could not investigate whether genetic factors affected the association between BCAA intake and outcome risks. Dietary BCAA intake was highly correlated with animal protein intake, and thus distinguishing between the effects of BCAA, meat, total protein and animal protein intakes was difficult. The causality of the relationship between BCAA intake and cardiovascular and metabolic diseases, and the associated mechanisms, are not yet fully understood.

In conclusion, our study findings suggest that higher dietary intakes of total BCAA and leucine were independently associated with a lower risk of all-cause mortality in our sample, and these associations were stronger in participants with higher serum TAG concentrations. More prospective studies in other populations and randomised clinical trials are needed to verify our findings and determine the underlying mechanisms.

\section{Acknowledgements}

Acknowledgements: The authors thank for the National Center for Health Statistics for providing the data. Financial support: The current study is supported by the National Natural Science Foundation of China (82173648), Innovative Talent Support Plan of the Medical and Health Technology Project in Zhejiang
Province (2021422878), Internal Fund of Ningbo Institute of Life and Health Industry, University of Chinese Academy of Sciences (2020YJY0212), Ningbo Clinical Research Center for Digestive System Tumors (Grant No. 2019A21003), Sanming Project of Medicine in Shenzhen (SZSM201803080) and Natural Science Foundation of Tianjin (20JCZDJC00540). Conflict of interest: There are no conflicts of interest. Authorship: All authors confirmed they have contributed to the intellectual content of this paper and have met the following four requirements: (a) significant contributions to the conception and design, acquisition of data or analysis and interpretation of data; (b) drafting or revising the article for intellectual content; (c) final approval of the published article and (d) agreement to be accountable for all aspects of the article thus ensuring that questions related to the accuracy or integrity of any part of the article are appropriately investigated and resolved. Ethics of buman subject participation: Not applicable.

\section{Supplementary material}

For supplementary material/s referred to in this article, please visit https://doi.org/10.1017/S1368980021004948

\section{References}

1. Liang YF, Long ZX, Zhang YJ et al. (2021) The chemical mechanisms of the enzymes in the branched-chain amino acids biosynthetic pathway and their applications. Biochimie 184, 72-87.

2. White PJ \& Newgard CB (2019) Branched-chain amino acids in disease. Science 363, 582-583.

3. Iwasaki M, Ishihara J, Takachi R et al. (2016) Validity of a selfadministered food-frequency questionnaire for assessing amino acid intake in Japan: comparison with intake from 4-day weighed dietary records and plasma levels. $J$ Epidemiol 26, 36-44.

4. Hu W, Sun L, Gong Y et al. (2016) Relationship between branched-chain amino acids, metabolic syndrome, and cardiovascular risk profile in a Chinese population: a cross-sectional study. Int J Endocrinol 2016, 8173905.

5. Harmon BE, Boushey CJ, Shvetsov YB et al. (2015) Associations of key diet-quality indexes with mortality in the multiethnic cohort: the dietary patterns methods project. Am J Clin Nutr 101, 587-597.

6. Hernandez JBR \& Kim PY (2021) Epidemiology Morbidity and Mortality. https://www.ncbi.nlm.nih.gov/books/ NBK547668/?report=classic (accessed May 2021).

7. Valerio A, D'Antona G \& Nisoli E (2011) Branched-chain amino acids, mitochondrial biogenesis, and healthspan: an evolutionary perspective. Aging 3, 464-478.

8. U.S. Department of Health and Human Services, Centers for Disease Control and Prevention \& National Center for Health Statistics (1994) Plan and operation of the Third National Health and Nutrition Examination Survey, 1988-94. Series 1: programs and collection procedures. Vital Health Stat $\mathbf{1}$, $1-407$.

9. Bialostosky K, Wright JD, Kennedy-Stephenson $\mathrm{J}$ et al. (2002) Dietary intake of macronutrients, micronutrients, 
and other dietary constituents: United States 1988-1994. Vital Health Stat 11, 1-158.

10. Alaimo K, McDowell MA, Briefel RR et al. (1994) Dietary intake of vitamins, minerals, and fiber of persons ages 2 months and over in the United States: Third National Health and Nutrition Examination Survey, Phase 1, 1988-1991. Adv Data (258), 1-28.

11. Han L, You D, Ma W et al. (2019) National trends in American Heart Association revised life's simple 7 metrics associated with risk of mortality among US adults. JAMA Netw Open 2, e1913131.

12. Orsini N, Li R, Wolk A et al. (2012) Meta-analysis for linear and nonlinear dose-response relations: examples, an evaluation of approximations, and software. Am J Epidemiol 175, 66-73.

13. Long L, Yang W, Liu L et al. (2020) Dietary intake of branched-chain amino acids and survival after colorectal cancer diagnosis. Int J Cancer 148, 2471-2480.

14. Shah SH, Sun JL, Stevens RD et al. (2012) Baseline metabolomic profiles predict cardiovascular events in patients at risk for coronary artery disease. Am Heart $J$ 163, 844-850.e841.

15. Du X, Li Y, Wang Y et al. (2018) Increased branched-chain amino acid levels are associated with long-term adverse cardiovascular events in patients with STEMI and acute heart failure. Life Sci 209, 167-172.

16. Noguchi Y, Zhang QW, Sugimoto T et al. (2006) Network analysis of plasma and tissue amino acids and the generation of an amino index for potential diagnostic use. Am J Clin Nutr $\mathbf{8 3}, 513 \mathrm{~s}-519 \mathrm{~s}$.

17. Cavallaro NL, Garry J, Shi X et al. (2016) A pilot, short-term dietary manipulation of branched chain amino acids has modest influence on fasting levels of branched chain amino acids. Food Nutr Res 60, 28592.

18. Le Couteur DG, Ribeiro R, Senior A et al. (2020) Branched chain amino acids, cardiometabolic risk factors and outcomes in older men: the concord health and ageing in men project. J Gerontol A Biol Sci Med Sci 75, 1805-1810.

19. Sun L, Hu C, Yang R et al. (2017) Association of circulating branched-chain amino acids with cardiometabolic traits differs between adults and the oldest-old. Oncotarget 8, 88882-88893.
20. Górska-Warsewicz H, Laskowski W, Kulykovets O et al. (2018) Food products as sources of protein and amino acids-the case of Poland. Nutrients 10, 1977.

21. Krajcovicova-Kudlackova M, Babinska K \& Valachovicova M (2005) Health benefits and risks of plant proteins. Bratisl Lek Listy 106, 231-234.

22. Børsheim E, Bui QU, Tissier S et al. (2008) Effect of amino acid supplementation on muscle mass, strength and physical function in elderly. Clin Nutr 27, 189-195.

23. Bifari F \& Nisoli E (2017) Branched-chain amino acids differently modulate catabolic and anabolic states in mammals: a pharmacological point of view. Br J Pharmacol 174, 1366-1377.

24. Okekunle AP, Zhang M, Wang Z et al. (2019) Dietary branched-chain amino acids intake exhibited a different relationship with type 2 diabetes and obesity risk: a metaanalysis. Acta Diabetol 56, 187-195.

25. Burrage LC, Nagamani SC, Campeau PM et al. (2014) Branched-chain amino acid metabolism: from rare Mendelian diseases to more common disorders. Hum Mol Genet 23, R1-R8.

26. Kitada M, Ogura Y, Monno I et al. (2019) The impact of dietary protein intake on longevity and metabolic health. EBioMedicine 43, 632-640.

27. Jackman SR, Witard OC, Philp A et al. (2017) Branched-chain amino acid ingestion stimulates muscle myofibrillar protein synthesis following resistance exercise in humans. Front Physiol 8, 390.

28. Jennings A, MacGregor A, Welch A et al. (2015) Amino acid intakes are inversely associated with arterial stiffness and central blood pressure in women. J Nutr 145, 2130-2138.

29. Grajeda-Iglesias C \& Aviram M (2018) Specific amino acids affect cardiovascular diseases and atherogenesis via protection against macrophage foam cell formation: review article. Rambam Maimonides Med J9, e0022.

30. Wang FH, Liu J, Deng QJ et al. (2019) Association between plasma essential amino acids and atherogenic lipid profile in a Chinese population: a cross-sectional study. Atherosclerosis 286, 7-13.

31. Yang $\mathrm{P}, \mathrm{Hu} \mathrm{W}, \mathrm{Fu} \mathrm{Z}$ et al. (2016) The positive association of branched-chain amino acids and metabolic dyslipidemia in Chinese Han population. Lipids Health Dis 15, 120. 